\title{
Mesothelioma in relation to asbestos exposure in laboratory work ${ }^{*}$
}

\author{
Penpatra Sripaiboonkij ${ }^{1 \#}$, Maritta S. Jaakkola ${ }^{2,3,4}$ \\ ${ }^{1}$ Faculty of Public Health, Thammasat University, Bangkok, Thailand; ${ }^{*}$ Corresponding Author: sripaiboonkijpor@Hotmail.com \\ ${ }^{2}$ Center for Environmental and Respiratory Health Research, University of Oulu, Oulu, Finland \\ ${ }^{3}$ Respiratory Medicine Unit, Institute of Clinical Sciences, University of Oulu, Oulu, Finland \\ ${ }^{4}$ Respiratory Medicine Unit, Oulu University Hospital, Oulu, Finland; maritta.jaakkola@oulu.fi
}

Received 17 March 2013; revised 20 April 2013; accepted 10 May 2013

Copyright (c) 2013 Penpatra Sripaiboonkij, Maritta S. Jaakkola. This is an open access article distributed under the Creative Commons Attribution License, which permits unrestricted use, distribution, and reproduction in any medium, provided the original work is properly cited.

\section{ABSTRACT}

Introduction: Mesothelioma is a malignant conditioncaused by exposure to asbestos that has typically a long latency period. The disease is usually found in the later stages when the prognosis is poor despite treatments. Objective: To report a mesothelioma found in a laboratory. Method: We reported here a case of a 60 yearold-man who was exposed to asbestos fibresfirst when he studied in a laboratory at high school and then he had exposure over the following years until graduating with a master's degree, but he did not have exposure after it. He was a smoker as a teenager and he gave up after 23 years. His first symptom was coughing at night which lasted for several months until the pleural fluid was discovered on the right side of thorax. This triggered investigations led to a diagnosis of mesothelioma. According to a systematic literature search, mesothelioma has not been reported previously in relation to asbestos exposure in laboratory work. An operation was carried out, but the patient refused to take chemotherapy due to the limited benefits that could be expected from it. Instead, he opted for a Budwig protocol. The case has had a good quality of life since the surgery, and did not have any medications. He has regular check-ups at the hospital. Conclusion: Our study has shown that

\footnotetext{
*Article focus: Mesothelioma in laboratory work.

Consent: An informed consent was obtained from the case for publication of this report and any accompanying images from his medical file. Conflicts of interest: The authors declare no conflict of interest.

Author contributions: PS: Interviewed case, discussion, summary new things that found from the case. MJ: Discussion, summary new things that found from the case.
}

mesothelioma is not only a risk to people directly exposed to asbestos, but also indirectly from, for example, laboratory work. People should avoid exposure to asbestos in daily life.

Keywords: Mesothelioma; Asbestos; Occupational Diseases; Laboratory; Budwig

\section{INTRODUCTION}

Mesothelioma is a malignant disease caused by exposure to asbestos fibres. It originates from the mesothelial lining of the pleura. The symptoms of the disease generally manifest 14 - 40 years after exposure to asbestos [14] which often leads to a late diagnosis as the cause is often not immediately apparent and early detection is rare. The disease is usually found in the later stages when the prognosis is poor despite the treatments.

Mesothelioma has previously been reported in connection with exposure to asbestos in asbestos mining, different types if insulation works, construction, and maintenance work [5-8], but in our systematic literature search, we did not find any previous reports linking mesothelioma to laboratory work.

Radiotherapy, chemotherapy and surgery are most frequently used treatments for mesothelioma, but these are all considered experimental as there is no standard treatment for it. Radiotherapy is a treatment option for mesothelioma, but has not proved to be unequivocally effective, and may adversely affect the surrounding organs leading to side effects [9].

Chemotherapy is another treatment method, but it often has limited success in treating mesothelioma [10-12]. Like radiation therapy, it may be used after surgery to eliminate any cancerous growth that could not be removed during the operation $[9,12]$. 
Budwig protocol is an alternative cancer treatment for patients who refuse the traditional cancer treatment methods mentioned. The idea behind this protocol is that a cancer cell has been proposed to have deficiencies of phosphatides and lipoproteins and these deficiencies result in a reduced level of oxygen in the cells [13]. The Budwig protocol is suggested to create a method to reoxygenate the cells. It includes flaxseed oil with cottage cheese and high intake of fruits, vegetables and fiber. Flaxseed oil provides linoleic and linolenic fatty acids, which are supposed to transfer oxygen into the cells, allowing for oxidation and detoxification of cellular waste. They also facilitate restoration of the lipid membrane of cells.

In this paper we report a case who was exposed to asbestos during his education in laboratory setting, while he had no exposure to any industrial setting. He did not encounter any asbestos later in his working career. We also present his experience with the alternative Budwig cancer treatment for which he opted instead of any traditional treatment.

\section{CASE PRESENTATION}

\subsection{Personal Characteristics and Work History}

The case is a 60-year-old man born in Poland who now works in a professional job at an academic institute in the UK. He was a smoker when he was 17 years old and he gave up on his birthday at 40 years old, having smoked about 20 cigarettes per day on average, giving cumulative exposure of 23-pack years.

The patient reported his first exposure to asbestos when he studied at high school from the age of 14 to 19 years old in the Technical College of Chemistry where he worked in laboratory classes which involved heating substances in glassware using gas burners. This required putting the glassware on meshes with asbestos to carry out tasks over a 4-year period. The meshes were kept in cupboards and, while handling them, there were clouds of asbestos due to dispersion during and after the heating process. After graduation from the college, he did a master degree in Chemical Engineering and Technology where he was further exposed to asbestos in laboratory. He might also have some asbestos exposure while he lived in the students' halls of accommodation, because there was asbestos in the roof and the roof was in poor condition.

\subsection{Medicalhistory}

The case was diagnosed with mesothelioma when he was 58 years old. The first symptom was the onset of coughing at night, which woke him up while he was on holidays in August 2010. He went to see a GP and was prescribed an antibiotic for a suspected infection but the symptoms did not improve. A few months later, the coughing symptoms at night worsened and another type of antibiotic was prescribed for suspected infection.

Six months later in 2011, the patient still had bad coughing episodes at night. After visiting a GP, the patient received another antibiotic. From auscultation of the lungs, the GP suspected the patient might have a buildup of fluid on the lung but did not refer the patient for any other test at the time. The coughing symptom did not improve after the patient completed the course of antibiotics over a 5 day period, so a chest x-ray was then taken. The results of the X-ray showed pleural fluid on the right side while the left lung was clear. Sprimetry showednormal values. The case was referred to a respiratory unit. He was suspected to have tuberculosis, respiratory infection or cancer. The patient had a computed tomography (CT) scan to investigate further. The X-rays showed thickening of the pleura and at least 2 litres of pleural fluidon the right side, but no findings in other parts of the body. 60 millilitres of fluid was taken from the pleura for analysis. A biopsy was also taken and cancer cells were discovered but the histological type remained unknown. A video-assisted thoracoscopy (VAT) was performed, which confirmed the diagnosis of mesothelioma with 1) a biopsy and 2) pleural fluid samples.

The patient chose not to have chemotherapy because he felt he was in good health and did not want to subject himself to the side-effects of such treatment, as chemotherapy has been shown to produce only minor improvement of lifespan for this kind of cancer.

The first operation was carried out on the case's right pleura by Keyhole surgery in September 2010. The operation team removed as much mesothelioma as possible. Following the operation, the patient had a short course of radiotherapy to try to prevent cancer cells from spreading to other parts of the body. Later control investigations showed that cancer had not spread.

The case had another consultation on chemotherapy treatment following completion of the radiotherapy. However, due to the limited benefits of this treatment against the side effects the case declined chemotherapy again. Instead he chose to take part in a clinical trial called ADAM which employsa treatment with pegylated arginine deiminase. However, the case was randomized in the control group.

The case was given PET (Positron Emission Tomography) and another CT scan (Computed Tomography). Before the PET/CT scan, the patient received an intravenous injection of radioactive glucose to help to assess the stage of cancer. At this stage, the mesothelioma was reported not to have spread and the patient was symptom-free. He reports to his GP monthly and is planned to 
have a CT every 2 months and a PET scan yearly. After the operation, the case has used the Budwig protocol diet. He has linseed oil and cottage cheese for his breakfast daily. Linseed oil contains a significant amount of omega-3 and omega- 6 fatty acids. Linseed oil and cottage cheese have been suggested to produce traces of arsenic which is believed to kill cancer cells but not healthy cells. He also eats fruit and vegetables during the day. To date, the case reported he is healthy.

\section{DISCUSSION}

Asbestos has been widely used in insulation and construction and to some extent also in vehicle brakes. It is known to be hazardous for people who are exposed to its fibres. The symptoms of diseases linked to asbestos exposure generally manifest 14 - 40 years after exposure to asbestos which often leads to a late diagnosis as the cause is often not immediately apparent [1-4].

The case presented here did not work in any occupations where workers traditionally come into contact with asbestos. However, he was directly exposed to material containing asbestos while he was a student in a laboratory setting. In our systematic Medline search we did not find any previous reports on asbestos exposure leading to significant health hazard in such conditions. Some studies have suggested that exposure to asbestos at younger ages may result in a shorter latency period for the disease [14] but traditionally, exposure to asbestos manifested as mesothelioma will take a long latency period.

Even though the health risks related to asbestos exposures are well known, asbestos is still widely used around the world, especially in Asian countries [15]. Epidemics of occupational diseases relating to asbestos have been reported in developing countries including Thailand recently [15-17]. Banning or limiting use of asbestos fibres worldwide will be an important issue to prevent further death and disability from such exposure. Our case indicates that even rather minor exposure may lead to a serious malignant disease, which mesothelioma inarguably is.

Mesothelioma is a malignant disease with poor prognosis with a rather short average survival time of 10.5 months [18] and an average 5-year survival rate of less than 9\% [19]. In patients treated with systemic chemotherapy, the median survival time (MST) is in the range of 6 to 12 months and the 2-year survival is <20\% [10]. When the case described in this report was first diagnosed with mesothelioma, he was given a life expectancy of less than 6 months. However; two years have now passed and he is still free of recurrence of this disease.

There is unsatisfactory evidence of chemotherapy providing real benefits in mesothelioma treatment [10-12]. Because of this our case chose an alternative cancer treatment, revolving around special dietary methods called
Budwig protocol. This seems to have worked well for our case, but randomized controlled trials are needed to evaluate if they can provide any long-term benefit for the patients with mesothelioma.

\section{CONCLUSION}

This case was diagnosed with mesothelioma at the age of 58 years. The only asbestos exposure he had experienced was in a laboratory setting while he studied in high school and it continued through to graduation with a master degree in Chemical Engineering. Two years after the diagnosis the case still has been free of disease, after his tumor was removed with surgery in September 2010. He did receive a short radiotherapy and has since then followed the Budwig protocol involving diet with flaxseed oil and high intake of fruit, vegetables and fiber. Diet may be an important consideration as complementary therapy for mesothelioma patients, but randomized clinical trials are needed before any firm conclusions can be made.

\section{ACKNOWLEDGEMENTS}

We would like to thank you the case who participated our study.

\section{REFERENCES}

[1] Bianchi, C., Giarelli, L., Grandi, G., Brollo, A., Ramani, L. and Zuch, C. (2005) Latency periods in asbestos-related mesothelioma of the pleura. European Journal Cancer Prevention, 6, 162-166.

[2] Robinson, B.W.S., Musk, A.W. and Lake, R.A. (2005) Malignant mesothelioma. The Lancet, 366, 397-408. doi:10.1016/S0140-6736(05)67025-0

[3] Okio, H., Kazu, S. and Masahiro, M. (2007) Diagnostic biomarker of asbestos-related mesothelioma: Example of translational research. Cancer Science, 98, 1147-1151. doi:10.1111/j.1349-7006.2007.00520.x

[4] Hino, O. and Maeda, M. (2008) Diagnostic tumor marker of asbestos-related mesothelioma. Environmental Health and Preventive Medicine, 13, 71-74. doi:10.1007/s12199-007-0014-9

[5] Fischbein, A., Luo, J.J. and Pinkston, R.G. (1991) Asbestosis, laryngeal carcinoma, and malignant peritoneal mesothelioma in an insulation worker. British Journalof Industrial Medicine, 48, 338-341.

[6] Muscat, E.J. and Wynder, L.E. (1991) Cigarette smoking, asbestos exposure, and malignant mesothelioma. Cancer Research, 51, $2263-2267$.

[7] Järvholm, B. and Sandén, Å. (1998) Lung cancer and mesothelioma in the pleura and peritoneum among Swedish insulation workers. Occupational and Environmental Medicine, 55, 766-770. doi:10.1136/oem.55.11.766

[8] Fonte, R., Gambettino, S., Melazzini, M., Scelsi, M., Zanon, C. and Candura, M.S. (2004) Asbestos-induced peritoneal mesothelioma in a construction worker. Environmental Health Perspectives, 112, 616-619. 


\section{doi:10.1289/ehp.6542}

[9] McAleer, M.F., Tsao, A.S. and Liao, Z. (2009) Radiotherapy in malignant pleural mesothelioma. International Journal of Radiation Oncology Biology Physics, 75, 326337. doi:10.1016/j.ijrobp.2009.06.037

[10] Mikulski, S. (1995) Treatment of malignant mesothelioma (review). International Journal of Oncology, 7, 1415-1420.

[11] Berghmans, T., Paesmans, M., Lalami, Y., Louviauk, I., Luce, S., Mascaux, C., et al. (2002) Activity of chemotherapy and immunotherapy on malignant mesothelioma: A systemic review of the literature with meta-analysis. Lung Cancer, 38, 111-121. doi:10.1016/S0169-5002(02)00180-0

[12] Waite, K. and Gilligan, D. (2007) The role of radiotherapy in the treatment of malignant pleural mesothelioma. Clinical Oncology, 19, 182-187. doi:10.1016/j.clon.2006.12.006

[13] Chamberlain, J. (2008) Cancer recovery guide: 15 alternative and complementary strategies for restoring health. Oxford, 68-72.

[14] Antman, K.H. (1993) Natural history and epidemiology of malignant mesothelioma. Chest, 103, 373S-376S. doi:10.1378/chest.103.4 Supplement.373S
[15] Park, E.K., Takahashi, K., Hoshuyama, T., Cheng, T.J., Delgermaa, V., Vinh Le, G. and Sorahan, T. (2011) Global magnitude of reported and unreported mesothelioma. Environmental Health Perspectives, 19, 514-518. doi:10.1289/ehp.1002845

[16] Joshi, T.K. and Gupta, R.K. (2004) Asbestos in developing countries: Magnitude of risk and its practical implications. International Journal of Occupational Medicine and Environmental Health, 17, 179-185.

[17] Yang, M. (2011) A current global view of environmental and occupational cancers. Journal of Environmental Science Health Part C: Environmental Carcinogenesis \& Ecotoxicology Reviews, 29, 223-249.

[18] Bagheri, R., Haghi, S.Z., Rahim, M.B., Attaran, D. and Toosi, M.S. (2011) Malignant pleural mesothelioma: Clinicopathologic and survival characteristic in a consecutive series of 40 patients. The Annals of Thoracic Cardiovascular Surgery, 17, 130-136. doi:10.5761/atcs.0a.09.01427

[19] Zarogoulidis, P., Orfanidis, M., Constadinidis, T.C., Eleutheriadou, E., Kontakiotis, T., Kerenidi, T., Kerenidi, T., Sakkas, L., Courcoutsakis, N. and Zarogoulidis K. (2011) A 26-year-old male with mesothelioma due to asbestos exposure: Case report. Medicine, 2011, Artical ID: 951732. doi:10.1155/2011/951732 